\title{
Effects of PKA-Mediated Phosphorylation of Snapin on Synaptic Transmission in Cultured Hippocampal Neurons
}

\author{
Pratima Thakur, ${ }^{1 \star}$ David R. Stevens, ${ }^{1 \star} \mathrm{Zu}$-Hang Sheng, ${ }^{2}$ and Jens Rettig ${ }^{1}$ \\ ${ }^{1}$ Physiologisches Institut, Universität des Saarlandes, 66424 Homburg/Saar, Germany, and ${ }^{2}$ Synaptic Function Unit, National Institute of Neurological \\ Disorders and Stroke, National Institutes of Health, Bethesda, Maryland 20892-4154
}

\begin{abstract}
Use-dependent activation of protein kinase A (PKA) modulates transmitter release, contributing to synaptic plasticity. Snapin, a PKA substrate in neurons, associates with the soluble $N$-ethylmaleimide-sensitive factor attachment protein receptor (SNARE) complex, and its phosphorylation leads to increased binding of synaptotagmin to the SNARE complex. We investigated the role of PKA-dependent phosphorylation of Snapin in hippocampal neurons. Overexpression of Snapin S50D, a mutant mimicking the phosphorylated state, resulted in a decreased number of readily releasable vesicles. In addition, both the release probability of individual vesicles and the depression rate during high-frequency stimulation were increased. Overexpression of Snapin S50A, a mutant that cannot be phosphorylated, did not alter the size of the pool or the probability of release. Furthermore, dialysis of Sp-cAMPS, a nonhydrolyzable analog of cAMP that will promote phosphorylation by PKA, also led to increased synaptic depression in cells overexpressing wild-type Snapin. These results establish Snapin as an important target of PKA in CNS synapses and indicate a role for Snapin in the plasticity of transmitter release.
\end{abstract}

Key words: calcium; hippocampus; neurotransmitter; synapse; presynaptic mechanism; SNARE complex

\section{Introduction}

Protein kinase A (PKA)-mediated phosphorylation underlies one form of synaptic facilitation (Greengard et al., 1991; Huang et al., 1994) by acting directly on proteins of the neurotransmitter release apparatus (Trudeau et al., 1996). Among the potential targets of protein kinase A is the recently discovered protein, Snapin, which associates with the soluble $N$-ethylmaleimide-sensitive factor attachment protein (SNAP) receptor (SNARE) complex (consisting of Syntaxin, SNAP-25 and vesicle-associated membrane protein 2) by binding directly to SNAP-25, consequently enhancing the association of the calcium sensor synaptotagmin with the SNARE complex (Ilardi et al., 1999). The C-terminal half of Snapin, and shorter peptides containing parts of this sequence, compete with Snapin binding to SNAP-25 in vitro. Injection of these peptides into presynaptic superior cervical ganglion neurons suppressed EPSP amplitude, indicating a role for Snapin in synaptic transmission by modulating the formation of functional SNARE complexes.

Snapin consists of 136 amino acids and can be phosphorylated by PKA at the serine residue located at position 50. Phosphorylation of Snapin at this serine resulted in a threefold increase in binding of Snapin to SNAP-25 (Chheda et al., 2001). Substitution of serine-50 with a negatively charged aspartate (S50D), a mutant

\footnotetext{
Received Feb. 19, 2004; revised May 10, 2004; accepted June 2, 2004.

This work was supported in part by the Deutsche Forschungsgemeinschaft (SFB 530/C9) to J.R. We thank Claudia Gerwin, Reiko Trautmann, and Carolin Bick for expert technical assistance.

*P.T. and D.R.S. contributed equally to this work.

Correspondence should be addressed to Jens Rettig, Physiologisches Institut, Universität des Saarlandes, Gebäude 59, 66424 Homburg/Saar, Germany. E-mail: jrettig@uniklinik-saarland.de. DOI:10.1523/JNEUROSCI.0590-04.2004

Copyright $\odot 2004$ Society for Neuroscience $\quad$ 0270-6474/04/246476-06\$15.00/0
}

that mimics the effect of phosphorylation, also resulted in increased affinity of Snapin for SNAP-25. Overexpression of Snapin S50D in adrenal chromaffin cells enhances secretion, whereas overexpression of the S50A mutant form, which is not a substrate for PKA, resulted in a modest decrease in secretion, suggesting that Snapin is involved in secretion in chromaffin cells and that phosphorylation modulates the efficacy of Snapin (Chheda et al., 2001).

Alterations in the efficacy of synaptic transmission underlying various forms of use-dependent synaptic plasticity have been described thoroughly in the hippocampus. Hippocampal "autapses" have proven particularly useful for the study of presynaptic events (Bekkers and Stevens, 1991). Here, we have further examined the role of PKA phosphorylation in the actions of Snapin by observing the effects of expression of S50D- and S50Amutated Snapin on transmission at autapses in cultured hippocampal neurons.

\section{Materials and Methods}

Hippocampal neuron cultures and electrophysiology. Micro-island culture preparation of hippocampal neurons was performed according to a modified version of published procedures (Bekkers and Stevens, 1991; Reim et al., 2001). After 10-14 d in culture, cells were infected with $50 \mu \mathrm{l}$ of activated Semliki Forest virus according to published protocols (Olkkonen et al., 1993; Ashery et al., 1999). Data acquisition and analysis were performed as described (Reim et al., 2001). Data are expressed as mean \pm SE. Statistical significance was tested by paired two-tailed Student's $t$ test.

All measurements were performed $6-12 \mathrm{hr}$ after infection. On the basis of Western blots, all three Snapin constructs were overexpressed to a similar level of $\sim 15$ - to 30 -fold over endogenous expression. Only dots containing a single neuron forming excitatory synapses (autapses) were 
used. Extracellular recording solution contained (in $\mathrm{mm}$ ): $172 \mathrm{NaCl}, 2.4$ $\mathrm{KCl}, 10$ HEPES, 10 glucose, $4 \mathrm{CaCl}_{2}, 4 \mathrm{MgCl}_{2}$, pH 7.3; 350 mOsm. Patch pipettes (2-3 M $\Omega$ ) were pulled from borosilicate glass (TWF 150; World Precision Instruments) on a Sutter puller and backfilled with (in $\mathrm{mm}$ ): $135 \mathrm{KCl}, 10$ HEPES, 1 EGTA, $4.6 \mathrm{MgCl}_{2}$, $4 \mathrm{Na}$-ATP, 15 creatine phosphate, $50 \mathrm{U} / \mathrm{ml}$ phosphocreatine kinase, $0.2 \mathrm{mg} / \mathrm{ml}$ neurobiotin, $\mathrm{pH} 7.3$; 315 mOsm. Currents were recorded with an EPC-9 amplifier driven by the Pulse 8.31 software package (HEKA Electronics, Lambrecht, Germany). EPSCs were elicited after short depolarizations of the membrane under voltage-clamp conditions. Such depolarizations elicit action potentials in the axon of the recorded cells and lead to synaptic transmission, which is recorded as EPSCs at the soma.

Series resistance was compensated $60-80 \%$; only recordings with access resistance below $10 \mathrm{M} \Omega$ were included in the analysis. Analysis was performed using IGOR Pro (Wave Metrics, Lake Oswego, OR).

Site-directed mutagenesis. S50A and S50D mutants were generated in a standard two-step megaprimer PCR using Taq polymerase with mutated oligonucleotides as primers and full-length Snapin complementary DNA as a template. The first step included two PCR reactions that span the mutation site. Final PCR products were cloned into the pGEX-4T vector (Amersham Biosciences, Freiburg, Germany). Mutations were further verified by DNA sequencing and then subcloned into the pSFV1 expression vector (Invitrogen, Bethesda, MD).

\section{Results}

We first studied the role of wild-type (wt) Snapin in synaptic transmission by overexpressing a Snapin-internal ribosomal entry site-green fluorescent protein construct in hippocampal neurons (see Materials and Methods). When stimulated at $0.2 \mathrm{~Hz}$, these neurons exhibited EPSCs of smaller amplitude $(2.48 \pm 0.42$ $\mathrm{nA} ; n=32)$ than EPSCs observed in control cells $(3.92 \pm 0.55 \mathrm{nA}$; $n=34 ; p<0.05$ ) (Fig. $1 A, B$ ). The postsynaptic currents measured during application of hypertonic sucrose, an indicator of the size of the readily releasable pool (RRP) (Rosenmund and Stevens, 1996), were slightly smaller in cells overexpressing Snapin, but this difference was not statistically significant. The integral of the current flowing during hypertonic sucrose treatment yields the charge flowing during this treatment. As shown in Figure 1, $C$ and $D$, the average charge transferred in control cells $(0.48 \pm 0.06 \mathrm{nC} ; n=12)$ was not significantly different from that measured in cells overexpressing the wild-type Snapin protein $[0.41 \pm 0.08 \mathrm{nC}(n=11) ; p>0.5]$. Summaries of these results are shown in Figure 1, $B$ and $D$, respectively. The release probability for controls $(0.05 \pm 0.01 ; n=11)$ was also not significantly altered compared with that in Snapin wt overexpressing neurons $(0.04 \pm 0.01 ; n=12)$ (see Fig. 7$)$. When tested at higher stimulation rates, EPSCs from both control and Snapin overexpressing cells showed similar degrees of depression at $10 \mathrm{~Hz}$ (Fig. $1 E$ ) and $20 \mathrm{~Hz}$ (Fig. 1 F). Control cells stimulated at $20 \mathrm{~Hz}$ initially showed facilitation that was absent in the Snapin overexpressing cells.

We then examined evoked EPSCs in neurons overexpressing the Snapin S50D mutant, which mimics the phosphorylated state of Snapin (Chheda et al., 2001). When stimulated at $0.2 \mathrm{~Hz}$, there was no significant difference in the EPSC amplitude of S50D overexpressing neurons $(3.29 \pm 0.46 \mathrm{nA} ; n=23)$ and those of controls (3.69 $\pm 0.45 \mathrm{nA} ; n=25)$ (Fig. $2 A, B)$. In contrast, the current recorded after application of hypertonic sucrose in S50D overexpressing cells was significantly reduced compared with that observed in control cells. The total charge flowing during hypertonic sucrose treatment in the S50D expressing cells $(0.29 \pm 0.04 \mathrm{nC} ; n=19)$ was $60.4 \%$ of that in control cells $(0.48 \pm 0.08 \mathrm{nC} ; n=26 ; p<0.05)$ (Fig. $2 C, D)$. This result indicates that the readily releasable pool is reduced in cells overexpressing the S50D mutant. We then calculated, on the basis of the measured RRP size, the probability of release required to
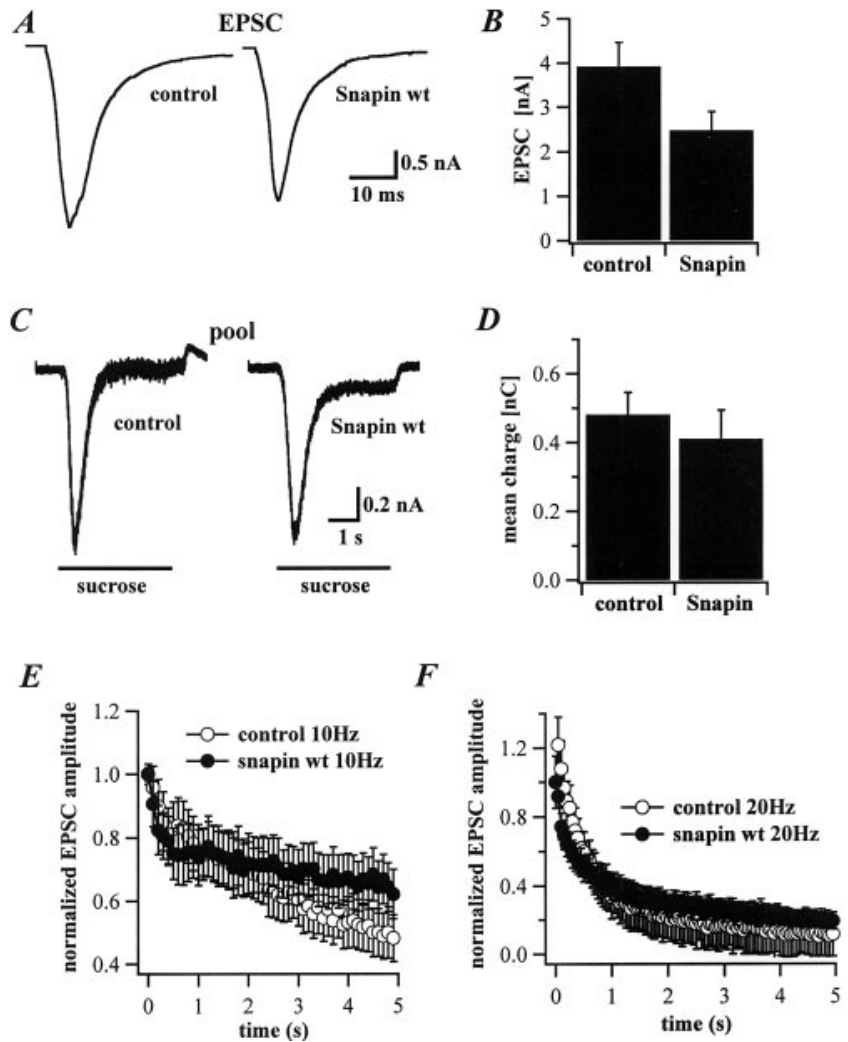

Figure 1. Overexpression of wild-type Snapin caused a reduction in basal synaptic transmission. $A$, EPSCs evoked in control and Snapin wt overexpressing neurons by $0.2 \mathrm{~Hz}$ stimulation. $B$, Histogram of the mean EPSC amplitude shows a modest reduction in the amplitude of the action potential evoked EPSCs in Snapin wt overexpressing neurons $(n=32)$ as compared with control ( $n=34 ; p<0.05)$. C, Postsynaptic currents induced by hypertonic sucrose recorded in control and Snapin wt overexpressing neurons. D, Histograms representing the RRP size determined as the mean of integral of charge transfer during hypertonic sucrose application in con$\operatorname{trol}(n=12)$ and Snapin wt overexpressing neurons $(n=11)$. The responses to trains of stimuli were examined to further test for effects on synaptic transmission. $E$, Stimulation control $(10 \mathrm{~Hz})$ $(\bigcirc), n=13$, and Snapin wt (O), $n=10$. F, Stimulation control $(20 \mathrm{~Hz})(\bigcirc), n=12$, and Snapin wt (O), $n=9$.

generate the observed EPSCs in the control and S50D cells. The mean probability of release for vesicles in cells overexpressing the S50D mutant was $0.10 \pm 0.01(n=19)$, whereas that in controls was $0.058 \pm 0.01(n=26 ; p<0.01)$ (see Fig. 7$)$.

Because the combination of smaller pool size and higher release probability favors synaptic depression during repetitive stimulation, we examined the responses of S50D overexpressing neurons at higher stimulus rates. When measured at $1 \mathrm{~Hz}$, the response of S50D cells remained similar to those of control cells (Fig. 3A), whereas at higher stimulus rates marked differences were observed. At stimulation rates of $\geq 2.5 \mathrm{~Hz}$, the S50D expressing neurons exhibited depression, whereas the control cells exhibited no change at $2.5 \mathrm{~Hz}$, facilitation at $5 \mathrm{~Hz}$, and depression at 10 and $20 \mathrm{~Hz}$ (Fig. 3B-E). The depression observed in control cells was preceded by a short period of facilitation, which was not observed in the S50D expressing cells (Fig. 3F). At a stimulation rate of $20 \mathrm{~Hz}$, the degree of depression in the control cells approached that observed in the S50D expressing cells. In all cases, the rate of depression was higher in the S50D cells.

We then examined the recovery from depression, because this provides an index of the refilling of the readily releasable pool. The stimulus protocol is shown in Figure $4 \mathrm{~A}$. After a stable period of responses at $0.2 \mathrm{~Hz}$ stimulation frequency, the cells were stim- 


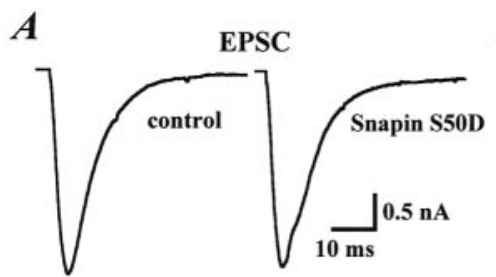

B

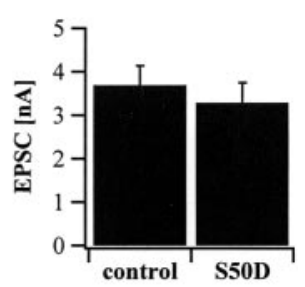

$C$

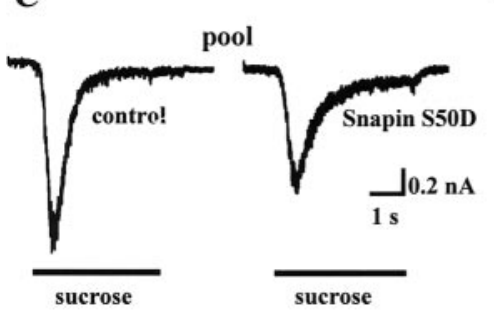

$\boldsymbol{D}$

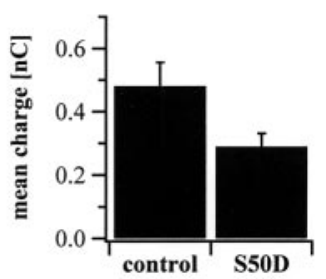

Figure 2. Overexpression of the Snapin S50D mutant leads to increased release probability. $A$, EPSC amplitude in control neurons and those overexpressing the Snapin S50D mutant. B, The mean amplitude of the EPSC in control and those overexpressing the S50D mutant were similar. C, The responses to hypertonic sucrose in control cells and in cells overexpressing the S50D mutant are shown. $D$, The mean released charge in neurons overexpressing the $S 50 D$ mutant was significantly less than that released by control cells $(p<0.05)$.

ulated at higher rates (see above). Recovery was followed with a stimulus rate of $1 \mathrm{~Hz}$ for $10 \mathrm{sec}$, after which the stimulation frequency was reduced to $0.2 \mathrm{~Hz}$. Neither the rate nor the degree of recovery was significantly different between the control and S50D overexpressing cells after stimulation at $2.5,5,10$, or $20 \mathrm{~Hz}$ (Fig. $4 B-E$ ). During the $1 \mathrm{~Hz}$ stimulation, all cells recovered and exhibited a period of augmentation before returning to a stable EPSC amplitude (Fig. 4F).

We compared the responses of neurons expressing the S50A mutant of Snapin with those of control neurons. The S50A mutant cannot be phosphorylated in vivo (Chheda et al., 2001) and will mimic the unphosphorylated state of Snapin. The EPSC amplitude during stimulation at $0.2 \mathrm{~Hz}$ was not different in control and S50A expressing neurons (for control: $3.57 \pm 0.51 \mathrm{nA}, n=$ 27; for Snapin S50A overexpressing neurons: $3.16 \pm 0.49 \mathrm{nA}, n=$ 23) (Fig. $5 A, B$ ). The amplitude of currents measured during hypertonic sucrose application was not significantly different in the S50A overexpressing cells when compared with controls (Fig. $5 C$ ), and the total charge transfer during hypertonic sucrose treatment was also similar in controls $(0.47 \pm 0.01 \mathrm{nC} ; n=12)$ and the S50A overexpressing neurons $(0.72 \pm 0.15 ; n=12)$ (Fig. $5 D$ ). As a consequence, the vesicle release probability was also not changed (for control: $0.06 \pm 0.01, n=27$; for Snapin S50A overexpressing neurons: $0.05 \pm 0.02, n=23$ ) (see Fig. 7). In contrast to the S50D overexpressing cells, the responses of S50A overexpressing cells to 10 and $20 \mathrm{~Hz}$ stimulation (Fig. $5 E, F$ ) were not different from those of controls. The S50A cells exhibited depression after an initial facilitation, as observed in controls.

To test further whether the phosphorylation state of Snapin contributes to the observed effects of expression of Snapin mutants on synaptic transmission, we tested the effects of overexpression of wild-type Snapin in the presence of Sp-cAMPS. The application of the cAMP analog Sp-cAMPS, which initiates the phosphorylation of Snapin by PKA in vivo (Chheda et al., 2001), leads to a buildup of phosphorylated Snapin and should have
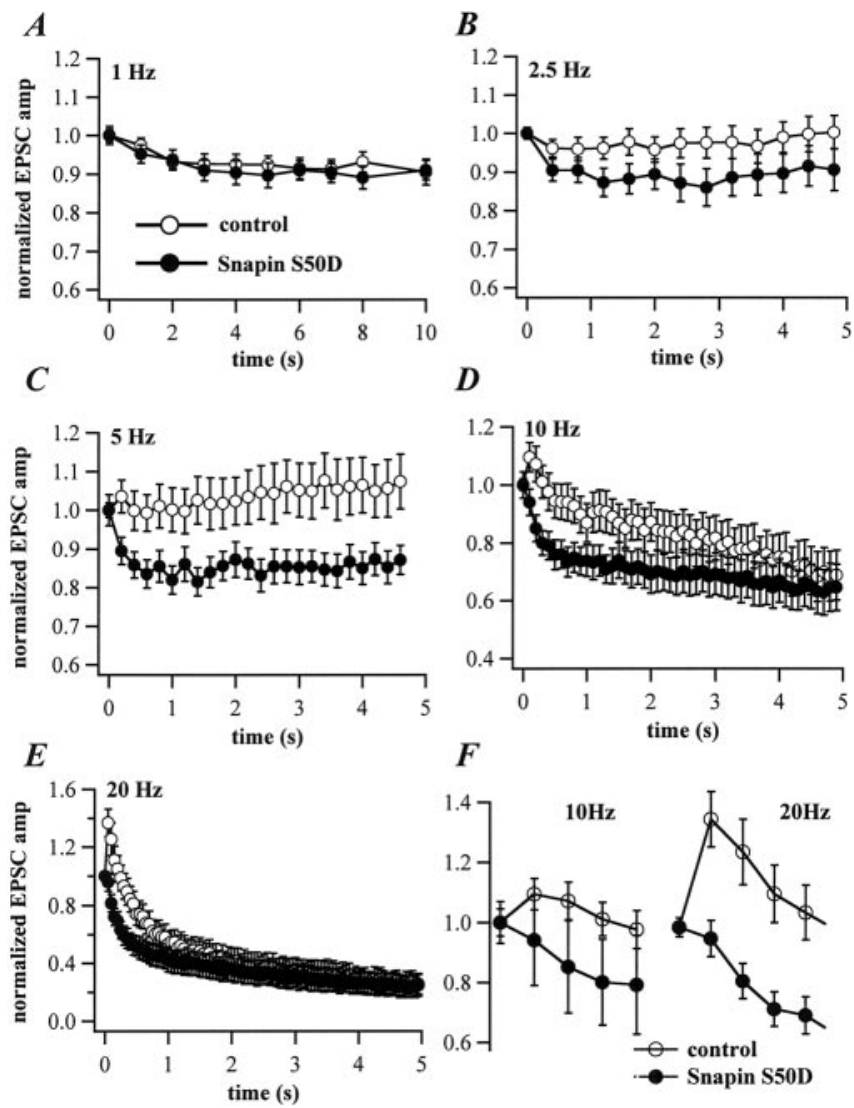

Figure 3. Snapin S50D overexpression alters short-term synaptic plasticity. A, Mean EPSC amplitude demonstrates facilitation followed by depression at higher stimulation rates in control $(\bigcirc)$ neurons. In neurons overexpressing Snapin S50D $(\bullet)$, the facilitation is absent and depression occurs at stimulation rates $>2 \mathrm{~Hz}$. A-E show comparison of mean EPSC amplitudes at rates of $1,2.5,5,10$, and $20 \mathrm{~Hz}$, respectively. $F$, Expanded graph showing the first four stimuli at 10 and $20 \mathrm{~Hz}$. The facilitation observed in control cells is clearly absent in the cells expressing the S50D Snapin.

effects similar to those of the S50D mutant. In contrast to the overexpression of wild-type Snapin alone, expression of wildtype Snapin in the presence of Sp-cAMPs did not alter EPSC amplitude (Fig. 6A,B). The mean amplitude of EPSCs in control cells recorded with Sp-cAMPS in the pipette $(1.72 \pm 0.25 \mathrm{nA} ; n=$ $17)$ and that in cells overexpressing the wild-type Snapin $(2.52 \pm$ $0.42 \mathrm{nA} ; n=12$ ) (Fig. $6 C$ ) were not significantly different after 9 min of recording in the whole-cell mode. Immediately after entering the whole-cell mode, the values were $2.25 \pm 0.29 \mathrm{nA}$ for control and $2.59 \pm 0.3 \mathrm{nA}$ for overexpressing neurons $(n=25)$ (Fig. 6B).

The calculated charge transfer during single EPSCs at a stimulus frequency of $0.2 \mathrm{~Hz}$ was $0.013 \pm 0.002 \mathrm{nC}(n=17)$ in control cells with Sp-cAMPS in the pipette. In contrast, in cells overexpressing wild-type Snapin, the transferred charge was $0.024 \pm$ $0.004 \mathrm{nC}(n=12 ; p<0.02)$ (Fig. $6 D)$ with Sp-cAMPS in the pipette. The charge transfer during hypertonic sucrose treatment was not significantly different (for control: $0.44 \pm 0.08 \mathrm{nC}, n=$ 17; for overexpressing neurons: $0.54 \pm 0.14 \mathrm{nC}, n=12$ ) (Fig. $6 E$ ). Calculation of the fractional release from the pool size and EPSC charge transfer values $(0.04 \pm 0.004$ for control and $0.07 \pm 0.01$ for overexpressing neurons; $p<0.01)$ confirmed that expression of Snapin in the presence of Sp-cAMPS led to increased release probability (Fig. 7). These results indicate that the overexpression of wild-type Snapin in the presence of Sp-cAMPS produces ef- 

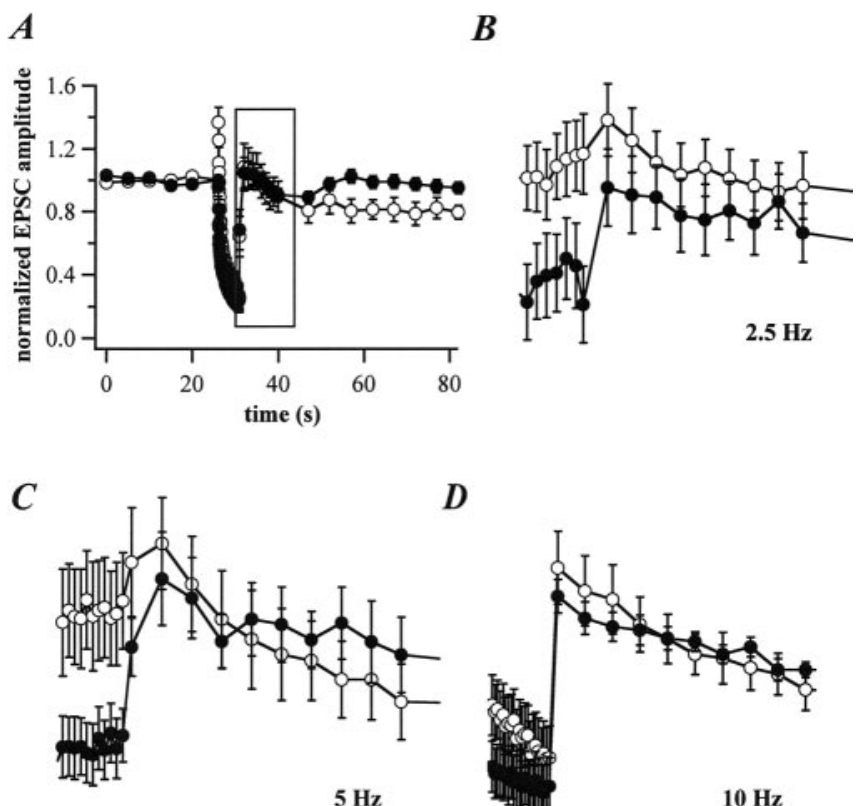

D
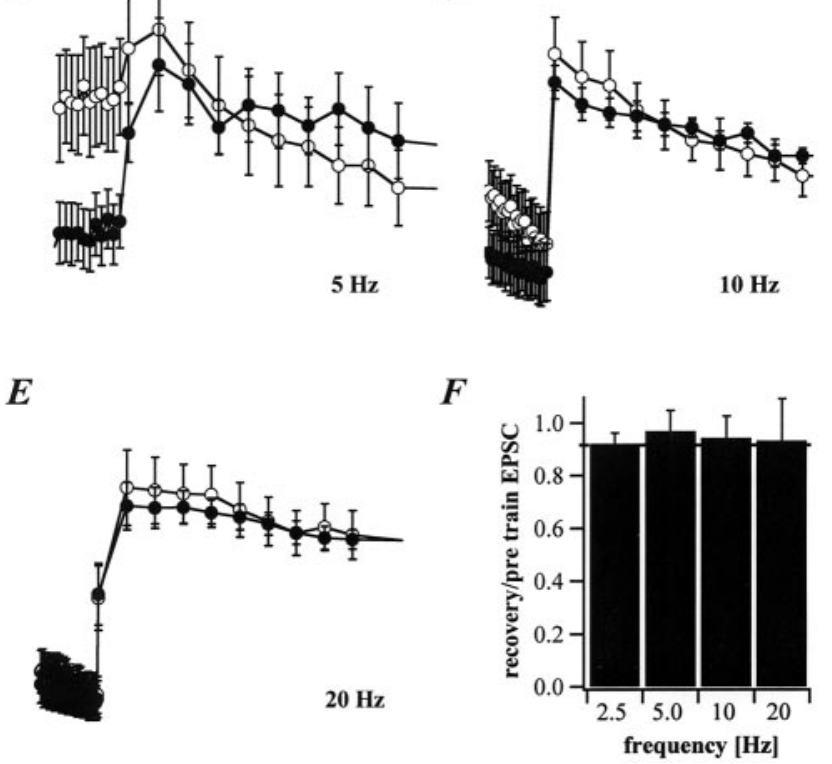

Figure 4. Recovery after depletion of the readily releasable pool is not altered in cells overexpressing the Snapin S50D mutant. A, Recovery from depletion was observed at a stimulus rate of $1 \mathrm{~Hz}$ after repetitive stimulation (O) (Snapin S50D overexpressing cells). Averaged responses during recovery from depression are shown for $2.5 \mathrm{~Hz}(B), 5 \mathrm{~Hz}(C), 10 \mathrm{~Hz}(D)$, and $20 \mathrm{~Hz}(E)$. The degree of recovery of Snapin S50D overexpressing cells was similar at all frequencies tested $(F)$.

fects similar to those observed in cells overexpressing Snapin S50D, consistent with the idea that phosphorylation modulates the action of Snapin. Comparison of the responses with highfrequency stimulation further supports this conclusion. During $10 \mathrm{~Hz}$ (Fig. 6F) and $20 \mathrm{~Hz}$ (Fig. 6G) stimulation, cells overexpressing Snapin in the presence of Sp-cAMPS exhibited more rapid depression than the control cells and showed no facilitation, in contrast to control cells. These results mimic the effects of the S50D Snapin mutant.

The main finding that phosphorylation of Snapin by protein kinase A increases vesicle release probability is summarized in Figure 7, where the release probabilities under the different experimental conditions are displayed.

\section{Discussion}

The above experiments were performed to characterize the general actions of Snapin and determine whether phosphorylation of Snapin by PKA regulates its function in neurotransmitter release. Our study showed that overexpression of Snapin in hippocampal neurons resulted in a modest decrease in EPSC amplitude, whereas the size of the readily releasable pool and the release probability were not significantly changed.

Cells overexpressing the S50D mutant of Snapin, in contrast,
$A$

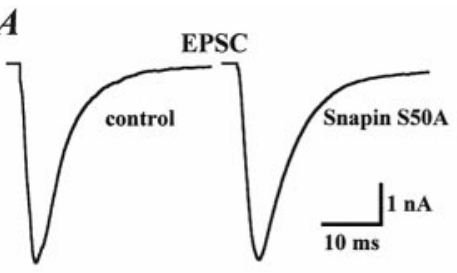

$\boldsymbol{B}$
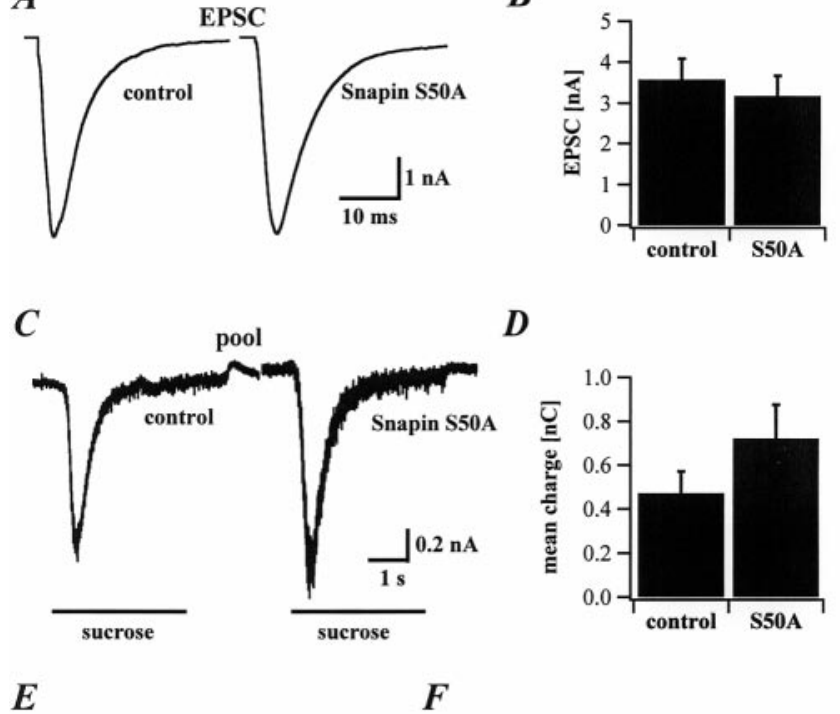

$D$

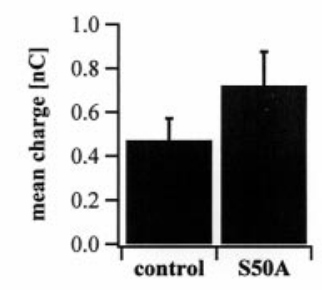

E
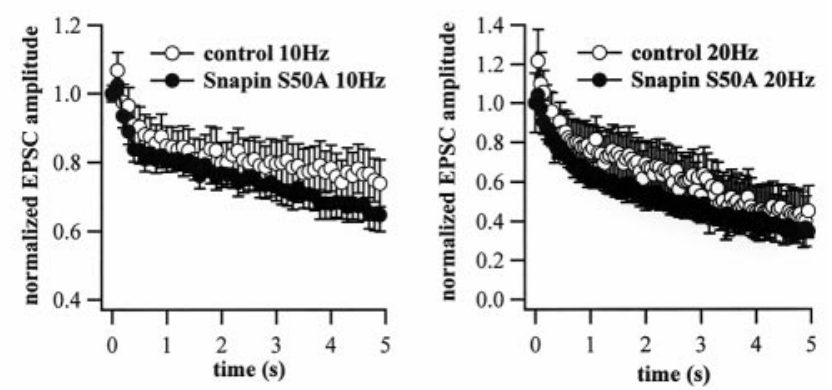

Figure 5. Overexpression of the Snapin S50A mutant reduced release probability. $A$, EPSCs in control and S50A overexpressing neurons. $B$, The mean EPSC amplitude in control neurons is similar to that observed in $\mathrm{S} 50 \mathrm{~A}$ overexpressing neurons. $C, A$ comparison of the response to hypertonic sucrose in control and Snapin S50A overexpressing neurons. D, The mean charge release in the control cells during hypertonic sucrose was not significantly different from that observed in cells overexpressing the Snapin S50A mutant. EPSC amplitude measured during stimulation at $10 \mathrm{~Hz}(E)$ and $20 \mathrm{~Hz}(F)$ in control cells $(O)$ did not differ from that in cells overexpressing the Snapin S50A mutant (O).

showed no reduction in EPSC amplitude but exhibited smaller responses to hypertonic sucrose, indicating a reduced readily releasable pool size. The probability of release must therefore be increased in these cells to overcome the smaller pool size or the EPSCs would decrease in size. Consistent with this finding is the observation that during repetitive stimulation the Snapin S50D mutant exhibited a stronger depression of EPSCs than did the wild-type or the S50A mutants, because an increase in release probability will favor synaptic depression (Zucker, 1989).

The finding that increasing wild-type Snapin reduces release probability whereas increasing Snapin S50D increases release probability could be attributable to the fact that the functional state of Snapin is regulated by PKA phosphorylation. In its phosphorylated state, Snapin could modulate synaptic transmission by enhancing release probability, whereas in its nonphosphorylated state, Snapin is inactive in regulating the release process. Thus, the negative actions of overexpression of wild-type Snapin on release could be readily explained by a dilution of endogenously functional Snapin in a phosphorylated state by overexpressed Snapin in an unphosphorylated state that overrides the phosphorylation capabilities of the cell.

We addressed this possibility by examining the effects of the Snapin S50A mutant. The S50A mutant cannot be phosphory- 

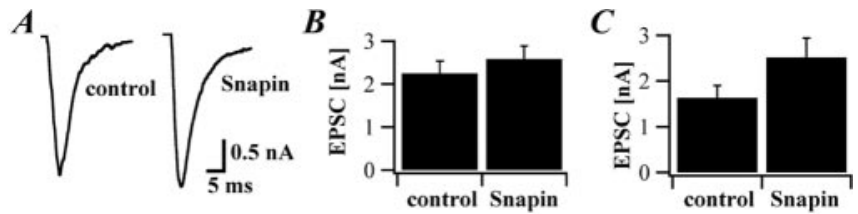

$\boldsymbol{D}$
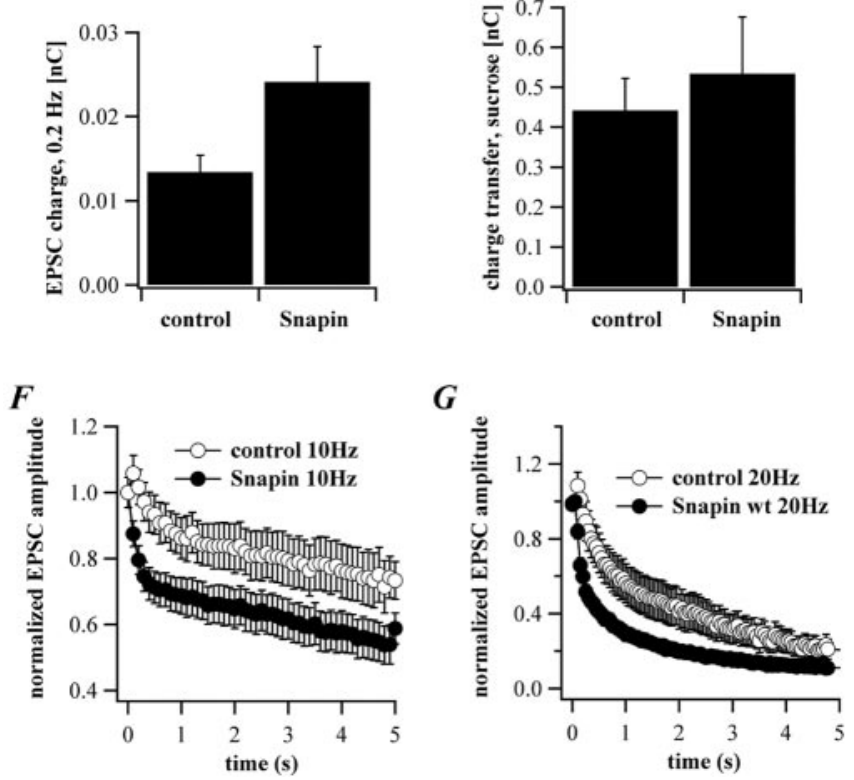

Figure 6. Overexpression of wild-type Snapin under conditions favoring phosphorylation does not alter EPSC amplitudes but increases vesicle release probability and synaptic depression. $A$, Representative traces of single EPSCS recorded from a control neuron and a neuron overexpressing wild-type Snapin. The pipette solution contained $1 \mathrm{~mm}$ Sp-cAMPS. B, Summary histograms of mean amplitude of 60 EPSCs in control cells or those overexpressing Snapin wt immediately after dialysis of $1 \mathrm{~mm}$ Sp-CAMPS. C, Summary histograms of mean EPSC amplitude in control $(n=17)$ and Snapin wt overexpressing neurons $(n=12) \sim 9$ min after dialysis of 1 mм Sp-CAMPS. D, Summary histograms of mean of charge transfer during $0.2 \mathrm{~Hz}$ stimulation in control ( $n=17)$ and Snapin wt overexpressing neurons $(n=12)$ on 1 mм Sp-CAMPS exposure; $p<0.02$. E, Summary histograms of mean charge transfer during hypertonic sucrose stimulation in control and in Snapin wt overexpressing neurons on $1 \mathrm{~mm}$ Sp-CAMPS exposure; $p>0.5$. $F$, Shown are averaged EPSC amplitudes during repetitive stimulation at $10 \mathrm{~Hz}$ in experiments in which the intracellular solution contained $1 \mathrm{~mm}$ Sp-CAMPS. Snapin wt overexpressing cells $(\mathbf{O})$ did not facilitate and exhibited more rapid depression than did the control neurons $(\bigcirc)$. G, A similar result was obtained at a stimulation rate of $20 \mathrm{~Hz}$. Note also that the facilitation seen at $20 \mathrm{~Hz}$ in control groups is reduced in this control group, which was performed with Sp-cAMPS in the pipette.

lated in vivo when expressed in neurons and lacks the negatively charged group at residue 50 found in the S50D mutant. Thus, the S50A mutant will function like the wild-type unphosphorylated Snapin. In addition, overexpression of this mutant will dilute the endogenously expressed and PKA-phosphorylated functional Snapin, negating the effects of endogenous kinase activity. As expected, we found that cells overexpressing the S50A mutant respond more similarly to cells overexpressing the wild-type Snapin than did Snapin S50D overexpressing neurons.

The fact that overexpression of Snapin S50D in hippocampal neurons leads to a decrease in the size of the readily releasable pool appears to be in contrast to the effect on exocytosis in adrenal chromaffin cells, in which an increase in the number of releasable vesicles was observed (Chheda et al., 2001). Chromaffin cells, however, release adrenalin and noradrenalin from large dense-core vesicles, whereas hippocampal neurons are a model

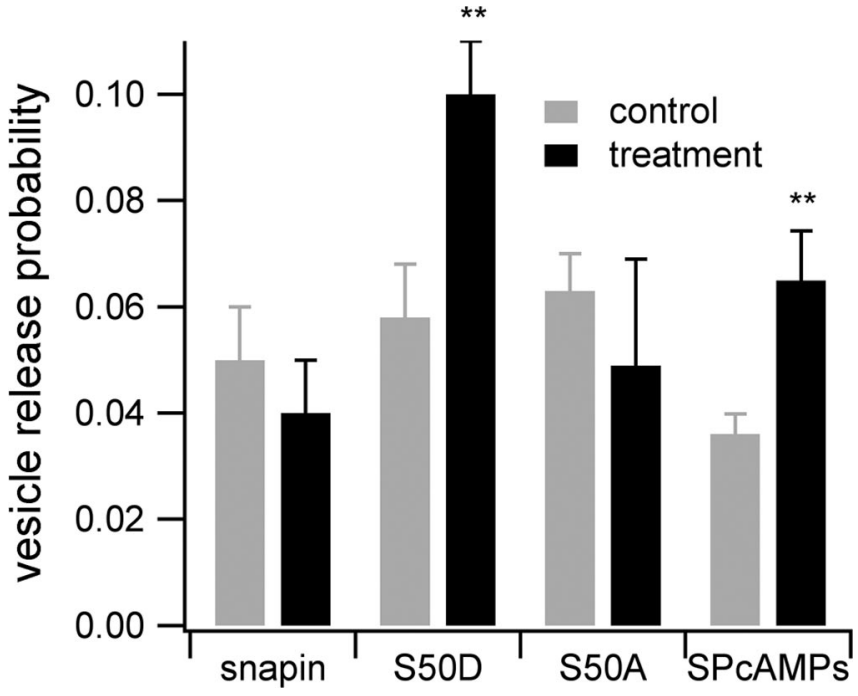

Figure 7. PKA phosphorylation of Snapin increases vesicle release probability. Summary histograms of average vesicle release probabilities for neurons overexpressing Snapin wt, Snapin S50D, Snapin S50A, and Snapin wt on $1 \mathrm{~mm}$ Sp-cAMPS exposure, compared with their respective controls. Asterisks indicate $p<0.01$.

system for release from small clear vesicles. Although these two classes of vesicles use a similar protein machinery to release their content into the extracellular space, many differences in the kinetic properties, such as rate constants, $\mathrm{Ca}^{2+}$ cooperativity, release probability, and pool sizes, have become apparent (Rettig and Neher, 2002). Most importantly, chromaffin granules reside in two different releasable pools (slowly releasable pool and RRP), whereas for small clear vesicles only one pool of releasable vesicles (RRP) has been identified. Therefore, the effects of Snapin phosphorylation on pool size in the two different model systems are not directly comparable.

Our results suggest a role for Snapin in neurotransmitter release and indicate that it is the phosphorylated form of Snapin that produces these effects. The results also indicate that phosphorylated Snapin is not an essential protein for synaptic transmission but rather functions as a modulator of release probability. Additional support for this proposed role of Snapin comes from the results of our experiments using Sp-cAMPS, an activator of cAMP-dependent PKA that increases the phosphorylation of Snapin in vivo. In the presence of Sp-cAMPS, overexpression of Snapin produced a similar increase in release probability compared with overexpression of the S50D mutant. In contrast, the reduction in pool size mediated by Snapin S50D overexpression was not observed in Sp-cAMPS-treated neurons overexpressing wild-type Snapin. This could be attributable to some long-term effects of Snapin S50D overexpression, which was present 6-12 hr before measurements, whereas Sp-cAMPS was applied transiently immediately before the measurements were performed. Alternatively, PKA activated by Sp-cAMPS most likely also phosphorylates proteins other than Snapin during the course of the experiments. It has been shown, for example, in adrenal chromaffin cells (Nagy et al., 2004), that PKA-phosphorylation of SNAP-25 leads to an increase in pool size that would counteract the negative effect of Snapin phosphorylation on pool size.

If the addition of Sp-cAMPS had not led to the phosphorylation of Snapin, we would expect a response similar to that of the wild-type Snapin overexpressing cells. Thus, the overexpression of wild-type Snapin under conditions that stimulate PKA phosphorylation in vivo would lead to changes similar to those in- 
duced by overexpression of the S50D mutant, which mimics phosphorylated Snapin. In the presence of Sp-cAMPS, cells overexpressing wild-type Snapin indeed demonstrated more depression, similar to cells overexpressing the S50D mutant. When SpcAMPS was included in the intracellular solution, the controls exhibited less facilitation at $20 \mathrm{~Hz}$. This result demonstrates that although Snapin is not the only target of PKA in the release machinery, enhanced phosphorylation in the absence of exogenously expressed Snapin leads to changes in release probability, which is consistent with the proposed role of PKA phosphorylation in the functional state of Snapin.

An increase in release probability leads to a higher degree of depression, because the readily releasable pool of vesicles is depleted more readily. Alternatively, depression may also occur if refilling of the readily releasable pool is hindered. We have observed no difference, however, in the refilling of this pool after induction of depression, indicating that the recruitment of vesicles into this pool is unchanged. In this case, the smaller size of the readily releasable pool is likely the result of the higher loss rate of vesicles from this pool caused by increased exocytosis, consistent with the estimated higher release probability; however, we cannot rule out the possibility that recruitment during high-frequency stimulation is changed, because this cannot be addressed by measurements involving glucose application.

Our results suggest a role for Snapin as a positive modulator in synaptic vesicle release. It appears that Snapin is more effective in the phosphorylated form. Thus, our study provides direct evidence in autapses of hippocampal neurons showing that Snapin is indeed a physiological modulator of synaptic transmission as well as a functional target of protein kinase A. It should be noted, however, that other presynaptic proteins have been shown to be phosphorylated by protein kinase A as well. For example, phosphorylation of the active zone protein Rab3-interacting molecule $(\mathrm{RIM} 1 \alpha)$ at serine 413 is responsible for the induction of presynaptic long-term potentiation (LTP) at parallel fiber synapses in the cerebellum (Lonart et al., 2003). The authors speculate that because this form of LTP is expressed by a multitude of other synapses such as hippocampal mossy fiber synapses and corticothalamic and corticostriatal synapses, the same mechanism of RIM1 $\alpha$ phosphorylation might also be responsible for LTP induction in these brain areas. Furthermore, Nagy et al. (2004) demonstrated recently that PKA phosphorylation of SNAP-25 at threonine 138 is required to maintain a large number of releaseready vesicles in adrenal chromaffin cells. In conjunction with a previous study in hippocampal neurons demonstrating the sensitivity of the PKA effect on Botulinum neurotoxin A (Trudeau et al., 1998), these data indicate a possible role for SNAP-25 in PKAdependent LTP. It is not clear which PKA target is the most influential determinant for the presynaptic induction of LTP in the hippocampus. Presumably, generation and interbreeding of phosphorylation-deficient knock-in mice of these proteins will help to settle this uncertainty. At present, our results provide a foundation for future studies to determine the physiological conditions under which Snapin is phosphorylated by PKA and to discover how the phosphorylation of Snapin modulates synaptic transmission via the cAMP-dependent signal transduction cascade.

\section{References}

Ashery U, Betz A, Xu T, Brose N, Rettig J (1999) An efficient method for infection of adrenal chromaffin cells using the Semliki Forest virus gene expression system. Eur J Cell Biol 78:525-532.

Bekkers JM, Stevens CF (1991) Excitatory and inhibitory autaptic currents in isolated hippocampal neurons maintained in cell culture. Proc Natl Acad Sci USA 88:7834-7838.

Chheda MG, Ashery U, Thakur P, Rettig J, Sheng ZH (2001) Phosphorylation of Snapin by PKA modulates its interaction with the SNARE complex. Nat Cell Biol 3:331-338.

Greengard P, Jen J, Nairn AC, Stevens CF (1991) Enhancement of the glutamate response by cAMP-dependent protein kinase in hippocampal neurons. Science 253:1135-1138.

Huang YY, Li XC, Kandel ER (1994) cAMP contributes to mossy fiber LTP by initiating both a covalently mediated early phase and a macromolecular synthesis-dependent late phase. Cell 79:69-79.

Ilardi JM, Mochida S, Sheng ZH (1999) Snapin: a SNARE-associated protein implicated in synaptic transmission. Nat Neurosci 2:119-124.

Lonart G, Schoch S, Kaeser PS, Larkin CJ, Südhof TC, Linden DJ (2003) Phosphorylation of RIM1 $\alpha$ triggers presynaptic long-term-potentiation at cerebellar parallel fiber synapses. Cell 115:49-60.

Nagy G, Reim K, Matti U, Brose N, Binz T, Rettig J, Neher E, Soerensen JB (2004) Regulation of releasable vesicle pool sizes by protein kinase A-dependent phosphorylation of SNAP-25. Neuron 41:417-429.

Olkkonen VM, Liljeström P, Garoff H, Simons K, Dotti CG (1993) Expression of heterologous proteins in cultured rat hippocampal neurons using the Semliki Forest virus vector. J Neurosci Res 35:445-451.

Reim K, Mansour M, Varoqueaux F, McMahon HT, Südhof TC, Brose N, Rosenmund C (2001) Complexins regulate a late step in $\mathrm{Ca}^{2+}$. dependent neurotransmitter release. Cell 104:71-81.

Rettig J, Neher E (2002) Emerging roles of presynaptic proteins in $\mathrm{Ca}^{++}$ triggered exocytosis. Science 298:781-785.

Rosenmund C, Stevens CF (1996) Definition of the readily releasable pool of vesicles at hippocampal synapses. Neuron 16:1197-1207.

Trudeau LE, Emery DG, Haydon PG (1996) Direct modulation of the secretory machinery underlies PKA-dependent synaptic facilitation in hippocampal neurons. Neuron 17:789-797.

Trudeau LE, Fang Y, Haydon PG (1998) Modulation of an early step in the secretory machinery in hippocampal nerve terminals. Proc Natl Acad Sci USA 95:7163-7168.

Zucker RS (1989) Short-term synaptic plasticity. Annu Rev Neurosci 12:13-31. 